\title{
Are there only Fold Catastrophes in the Diels-Alder Reaction between Ethylene and 1,3-Butadiene?
}

\author{
Leandro Ayarde-Henríquez, ${ }^{* a}+$ Cristian Guerra, ${ }^{a}$ Mario Duque-Noreña ${ }^{a}$, Elizabeth Rincón ${ }^{b}$, Patricia \\ Pérez, ${ }^{a}$ and Eduardo Chamorro*a+ \\ This work revisits the topological characterization of the Diels-Alder reaction between 1,3-butadiene and ethylene. In \\ contrast to the currently accepted rationalization, we here provide strong evidence in support of a representation in terms \\ of seven structural stability domains separated by a sequence of 10 elementary catastrophes, but all only of the fold type, \\ i.e., $\mathrm{C}_{4} \mathrm{H}_{6}+\mathrm{C}_{2} \mathrm{H}_{4}$ : 1-7- $[\mathrm{FF}] \mathrm{F}\left[\mathrm{F}^{\dagger} \mathrm{F}^{\dagger}\right]\left[\mathrm{F}^{\dagger} \mathrm{F}^{\dagger}\right][\mathrm{FF}] \mathrm{F}^{\dagger}-0: \mathrm{C}_{6} \mathrm{H}_{10}$. Such an unexpected finding provides fundamental new insights \\ opening simplifying perspectives concerning the rationalization of the $\mathrm{CC}$ bond formation in pericyclic reactions in terms of \\ the simplest Thom's elementary catastrophe, namely the one-(state) variable, one-(control) parameter function.
}

\section{Introduction}

The mechanism of the Diels-Alder (DA) reaction will continue to be a subject of major concern and key importance in chemistry. ${ }^{1}$ The study of this reaction has contributed to the development of the entire field of chemistry with a broad impact. ${ }^{2}$ Considered to be a prototypical example of pericyclic processes, ${ }^{3}$ the elucidation of the nature of what is the exact meaning of a "concerted" mechanism4 is far away to be concluded. ${ }^{3 a, 5}$ At the heart of controversy and debate remains the characterization of the bonding nature featuring the transition structures as well as the electron rearrangement along the entire chemical transformation. ${ }^{5 a}$, 5c, 5g, 6 Orbital-based approaches, ${ }^{2 c,} 3 b, 7$ conceptual descriptors derived from the density functional theory framework, $5 \mathrm{~d}, 8$ and topological-defined quantum approaches, ${ }^{5 e-g}$, 9 have been applied to obtain insights concerning the intimate details of this key one-step reaction mechanism.

The topological analysis of quantum mechanical local functions ${ }^{10}$ provides a partition of the 3D molecular space into basins of attractors (maxima) that can be associated to chemically meaningful information and/or concepts. Such an analysis provides topologically derived structures defined in terms of the associated critical points (CPs) and their interconnections via gradient paths. Within this perspective of a quantum chemical topology framework featuring the concept of gradient vector fields, the analysis and characterization of the gradient field of the electron density itself (i.e., the quantum theory of atoms in molecules QTAIM $^{10 d, 11)}$ and the electron

\footnotetext{
a. Universidad Andres Bello. Facultad de Ciencias Exactas. Departamento de Ciencias Químicas. Avenida República 275, 8370146, Santiago. Chile.

b. Facultad de Ciencias, Instituto de Ciencias Químicas, Universidad Austral de Chile, Las encinas 220, 5110033, Valdivia, Chile.

† Corresponding author e-mails: I.ayardehenriquez@uandresbello.edu (L.A-H); echamorro@unab.cl (EC)

localization function (ELF10e, 10f, 12) constitute nowadays key valuable methodological approaches for the interpretation of chemical bonding and reactivity. ${ }^{13}$ Several other functions have been explored within such a chemically oriented topological
}

framework, including among others the Laplacian of density, ${ }^{14}$ the nuclear potential, ${ }^{15}$ the electrostatic potential, ${ }^{16}$ the virial field, ${ }^{17}$ the magnetically induced molecular current distribution, ${ }^{18}$ and even the spin density. ${ }^{19} \mathrm{~A}$ description of the change associated to the topologically defined molecular structures as response to the variation of control parameters can be addressed via the theory of elementary catastrophes, 20 as it has been mainly exploited within both the QTAIM ${ }^{10 d}$ and ELF10e, 10f, 12 frameworks. Within the so-called bonding evolution theory (BET), ${ }^{21}$ changes in the topology of ELF along a chosen reaction path (e.g. IRC22) are characterized in terms of Thom's elementary catastrophes. ${ }^{20,23}$ Such a model for studying the evolution of the rearrangement of electron pairing along the reaction coordinate, and hence, chemically significant events (including bond making/breaking processes), become naturally associated to specific structural stability domains (SSDs) separated by catastrophe bifurcations. ${ }^{20,23}$ Any chemical reaction can be in such a way represented in terms of a precise sequence of catastrophic bifurcations associated to electron pair topologies that enables a straightforward rationalization or interpretation of the evolution of bonding patterns. ${ }^{24}$ It should be strongly emphasized however that such correlation between ELF and chemical bonding is of course just topological, and not energetical. BET has provided meaningful insights on an everincreasing number of reactive processes related to problems in almost all fields of chemistry, ${ }^{24 c, 24 d}$ including, for instance, key questions on bonding and reactivity related to the activation of $\mathrm{C}-\mathrm{H}$ bonds, ${ }^{25}$ proton/hydrogen transfer reactions, ${ }^{26}[4+2]$ cycloadditions, $^{9,} \quad 27 \quad[3+2]$ cycloadditions, $^{28}[1,3]$ dipolar cycloadditions, ${ }^{9,} 29$ the process of fixation of $\mathrm{CO}_{2}$ by metal complexes, ${ }^{30}$ decarbonylation of unsaturated cyclic ketones, ${ }^{31}$ the nature of phase transitions for the group IV elements, ${ }^{32}$ the formation of hemiaminals, ${ }^{33}$ Cope, ${ }^{9,} 34$ and Claisen ${ }^{35}$ rearrangements, the thermal decomposition of $\alpha$-ketoesters, ${ }^{36}$ hydrometallation of acetylene, ${ }^{37}$ oxidative additions of ammonia to pincer complexes, ${ }^{38}$ the Curtis rearrangement, ${ }^{39}$ the catalytic Noyori hydrogenation, 40 and the Wittig reaction. ${ }^{5 a}$ In such a context, the suitability of the characterization of the local character of the local ELF function dependents on a proper identification of the associated elementary catastrophes, and 
hence, the analysis of how the equilibria of ELF change as the control parameters changes. That is precisely the attractive simplification underlying any effort concerning the application of the elementary catastrophe theory. ${ }^{20,23}$

A BET analysis of the prototypical DA reaction between ethylene and 1,3-butadiene has been reported based on the B3LYP/6$31 \mathrm{G}(\mathrm{d})$ level of theory. ${ }^{\mathrm{e}}$, $5 f$ These results provided a representation in terms of seven SSDs separated by a sequence of ten elementary catastrophes of fold $\left(\mathrm{F}\right.$ and $\mathrm{F}^{+}$) and cusp ( $\mathrm{C}$ and $\mathrm{C}^{+}$) type, i.e., $\mathrm{C}_{4} \mathrm{H}_{6}+\mathrm{C}_{2} \mathrm{H}_{4}: 1-7-[\mathrm{CC}] \mathrm{C}\left[\mathrm{F}^{\dagger} \mathrm{F}^{\dagger}\right]\left[\mathrm{F}^{\dagger} \mathrm{F}^{\dagger}\right][\mathrm{CC}] \mathrm{C}^{+}-0$ : $\mathrm{C}_{6} \mathrm{H}_{10}$, with eight bifurcations occurring simultaneously. ${ }^{5 f}$ In principle, the identification of the catastrophe should be achieved by examining the nature of CPs at the configuration of nuclei that constitute the bifurcation point. Degenerate critical points lead to catastrophes, if some conditions are satisfied. ${ }^{20}$, 23 In practical implementations reported until now, the system is examined in terms of configurations close enough to such a bifurcation state which might yield an imprecise characterization of the catastrophe. Certainly, a formal analysis will require the examination of the behavior of the determinant of the Hessian matrix as well as the associated mobility of CPS of ELF along the reaction path. Our goal stress the fact that in order to obtain significant results, this original methodology should be applied. This is precisely the conceptual novelty of the present work. Following ongoing interests in to achieve a better rationalization concerning the process of identifying the nature of catastrophe bifurcations within the conceptual framework provided by the elementary catastrophe theory, 20,23 we revisit such a prototypical key DA reaction mechanism. A careful examination of the behavior of the CPs that are implied in each bifurcation, will provide as discussed in detail, new and significant results.

\section{Theoretical details}

Let us in this point recall just only some formal aspects of the bonding evolution theory framework. It refers as mentioned to the combined use of the topological analysis of ELF ${ }^{10 e}, 10 f, 12$ and the Thom's catastrophe theory ${ }^{20}$ to the study of the evolution of a reaction system along a given pathway. It has been indeed argued that BET provides a clear demarcation between chemical and nonchemical processes. ${ }^{5 a}$, 6a, 24a, 24c, 24d Moreover, the BET framework based on the ELF topological description provides a universal language for chemical bonding that is formally supported on the rigorous mathematical terminology arising from the topological theory of dynamical systems. ${ }^{23 \mathrm{~b}, 41}$ Given any electronic state for a N-electron molecular system represented in terms of a single determinant of Hartree-Fock or Kohn-Sham molecular orbitals $\psi_{i}(\boldsymbol{r} ; \boldsymbol{R})$ with occupation numbers $n_{i}$, the ELF $\eta(\boldsymbol{r} ; \boldsymbol{R})$ is a useful relative measure of the electron pair localization characterizing the corresponding electron density distribution $\rho(\boldsymbol{r} ; \boldsymbol{R})=\sum_{i}^{N} n_{i}\left|\psi_{i}(\boldsymbol{r} ; \boldsymbol{R})\right|^{2}$. The notation $(\boldsymbol{r} ; \boldsymbol{R})$ stands for solutions concerning the space coordinate for electrons $\boldsymbol{r}$ at fixed nuclei space coordinates $\boldsymbol{R}$. Note that within the framework of DFT, ELF is a property based directly on the electron density, able to be interpreted in terms of the positive-definite local Pauli $\tau_{P}(\boldsymbol{r} ; \boldsymbol{R})$ and Thomas Fermi, $\tau_{h}(\boldsymbol{r} ; \boldsymbol{R})$, kinetic energy densities in the given system, namely, ${ }^{10 e}, 42 \eta(\boldsymbol{r} ; \boldsymbol{R})=\left[1+\left[\tau_{P}(\boldsymbol{r} ; \boldsymbol{R}) / \tau_{h}(\boldsymbol{r} ; \boldsymbol{R})\right]^{2}\right]^{-1}$. As it is well known, $10 \mathrm{e}, 42$ the ratio $\tau_{P}(\boldsymbol{r} ; \boldsymbol{R}) / \tau_{h}(\boldsymbol{r} ; \boldsymbol{R})$ provides the key information on the relative local excess of kinetic energy density associated to the Pauli principle, given we have that $\tau_{P}(\boldsymbol{r} ; \boldsymbol{R})=$ $\sum_{i}^{N}\left|\nabla \psi_{i}(\boldsymbol{r} ; \boldsymbol{R})\right|^{2}-(1 / 8)|\nabla \rho(\boldsymbol{r} ; \boldsymbol{R})|^{2} / \rho(\boldsymbol{r} ; \boldsymbol{R})$ and $\tau_{h}(\mathbf{r} ; \mathbf{R})=$ $(3 / 5)\left(6 \pi^{2}\right)^{2 / 3} \rho(\mathbf{r} ; \mathbf{R})^{5 / 3} . \eta(\boldsymbol{r} ; \boldsymbol{R})$ takes values in the range $[0,1]$, the highest values being associated to the spatial positions $\boldsymbol{r}$ with higher electron localization (as compared to the arbitrarily chosen uniform electron gas reference). ${ }^{10 e}, 42$ Currently, both single-determinantal and correlated wave functions can be analysed using extended formulations of the electron localization function. $28,{ }^{43} \mathrm{~A}$ way to obtain the ELF in excited states of any symmetry have been proposed within the linear-response time-dependent density functional theory framework. ${ }^{44}$

The analysis of the gradient vector field of ELF, $\nabla_{r} \eta(\boldsymbol{r} ; \boldsymbol{R})$, provides a division of the molecular space $\rho(\boldsymbol{r} ; \boldsymbol{R})$ into basins of attractors that are, empirically, associated to chemically meaningful concepts such as atomic cores, valence bonds, lone pairs, and other elements of chemical bonding. ${ }^{13 b, 45}, 29$ The CPs of $\eta(\boldsymbol{r} ; \boldsymbol{R})$ and their connectivity determine the ELF molecular graph of the system, providing a powerful interpretative model providing important and valuable information about bonding and reactivity in chemical processes. ${ }^{10 e, 10 f, 12}$ In such a picture of bonding, the valence basin densities are delocalized throughout those associated to the inner atomic shell densities of core basins. ${ }^{10 e, 10 f, 12}$ The valence basins are characterized by the number of core basins with which they have a boundary. ${ }^{13 b}$ Valence monosynaptic basins can be associated for instance to electron lone pairs, whereas disynaptic basins are associated to typical two-centre bonds. Such a synaptic order provides a useful way to represent multicentre bonds within a topological framework of rationalization of chemistry. ${ }^{13 b}$ The $\eta(\boldsymbol{r} ; \boldsymbol{R})$ is a real-valued smooth function on $\mathbb{R}^{3}$ having all types of CPs, i.e., $(3,-3)$ : attractors or maxima of index $0,(3,-1)$ : saddles of index $1,(3,+1)$ : saddles of index 2 , and $(3,+3)$ : repellors or minima of index 3 . There is no harm in alternatively defining the index of a $\mathrm{CP}$ as the number of positive eigenvalues of its Hessian. ${ }^{46}$ The well-known notation in terms of (rank, signature) of a CP is above indicated. ${ }^{10 a}$, 10d, 21 The mathematical concepts of Thom's catastrophe theory can be therefore applied to the analysis of the gradient dynamical system $\dot{\boldsymbol{r}}=-\nabla_{\boldsymbol{r}} \eta(\boldsymbol{r} ; \boldsymbol{R})$ (i.e., the gradient vector field of the local function ELF, where the dot over the $\boldsymbol{r}$ stands for time derivative). The behavior of such a gradient system become uniquely defined by the equilibrium condition $\nabla_{\boldsymbol{r}} \eta(\boldsymbol{r} ; \boldsymbol{R})=0$. Within such a context, a subset of nuclei space coordinates $\boldsymbol{R}$ can be indeed considered as the external control parameters, whereas the electronic space coordinates $\boldsymbol{r}$ define the internal state variables, i.e., a subject to perturbations from the external variables. The points satisfying the equilibrium condition are CPs, whose connectivity determines the molecular graph at the fixed nuclei position. The CPs of $\eta$ corresponds to an equilibrium of the system. The evolution of the equilibria (i.e., the stationary state of CPs at a given fixed $\boldsymbol{R}$ ) is determined by examining the determinant of the Hessian matrix $H_{i j}=\partial \eta(\boldsymbol{r} ; \boldsymbol{R}) / \partial r_{i} \partial r_{j}$ for CPs at each 
configuration (i.e., fixed $\boldsymbol{R}$ ). If the determinant of the Hessian matrix at any CP of $\eta(\boldsymbol{r} ; \boldsymbol{R})$ is equal to zero, or in practical applications show a strong tendency to reach this value, such $\mathrm{CP}$ is said to be degenerate or non-hyperbolic, otherwise it is of the Morse-type or hyperbolic. 20,23 The set of configurations of the control space for which the Hessian matrix of a given CP is nonzero defines the domain of stability of the critical point. The system evolves from a domain of stability to another upon small changes in the control parameters $\boldsymbol{R}$. If none of CPs of the system change, the system is said to be located within a given SSD, which is associated to a bonding state of the system. An essential fact is that the number of Morse type CPs in each SSD always satisfies the Poincaré-Hopf theorem. ${ }^{47}$ Henceforth, the change of the nuclear space coordinates (i.e., along the chosen pathway) will determine topological changes implying different SSDs which are (conceptually) associated to the change in bonding patterns along the chemical transformation represented along the chosen pathway. Bifurcation catastrophes (i.e., a change in the topographic map induced by the splitting of a non-hyperbolic CP into a finite collection of isolated hyperbolic CPs) separates different SSDs. That is the basic hypothesis of such a methodological framework, and it is, of course, based on the assumption of validity of the Thom's conjecture, 20, 23 which determines the change of the potential function upon small variations of the control parameters. The identification of the catastrophe is achieved by examining the nature of the CPs at the configuration of nuclei that constitute the bifurcation point $\left(\boldsymbol{r}^{*}, \boldsymbol{R}^{*}\right)$. Thom's theory predicts that, under suitable conditions, 20,23 the local character of potentials in the neighborhood of a degenerate CP can be explored through canonical polynomic forms in 1- or 2- state variables, i.e., the so-called elementary catastrophes, i.e., fold, cusp, swallow tail, hyperbolic umbilic, elliptic umbilic, butterfly, and parabolic umbilic. ${ }^{20,23}$ So far, and within the BET perspective, only cusp, fold, and elliptic umbilic elementary catastrophes have been found to be fingerprints associated with the elementary processes driven a chemical transformation. We here stresses that only a precise monitoring of the evolution of the CPs of $\eta(\boldsymbol{r} ; \boldsymbol{R})$ along a reaction path, i.e., following changes in the number and type of all CPs and hence, the determination of the exact form of the universal unfolding, ${ }^{20,23}$ will enable the proper association of bifurcation catastrophes to the key breaking/forming chemical bonding events. The unfoldings are simply parametric polynomials of degree equal or greater than 3 in virtue of which is possible to approximate the function $\eta(\boldsymbol{r} ; \boldsymbol{R})$ in the locality of a topological bifurcation, after fulfilment of specific requirements. ${ }^{41,48}$ The set of external parameters has the effect of splitting the non-hyperbolic CP into a new set of Morse CPs, i.e., a collection of finite and isolated non-degenerate CPs. As a result, degeneracy is overcome. Henceforth the evolution of the determinant of the Hessian matrix for CPs of the $\eta(\boldsymbol{r} ; \boldsymbol{R})$ along a reaction path provides always a strong and suitable tool for identifying when a CP will become a non-hyperbolic or a degenerate one.

\section{Computational details}

Following Andrés et al. ${ }^{5 f}$ findings, all calculations for the DA reaction were reproduced at B3LYP/6-31G(d) DFT level of theory using the Gaussian 09 package of programs. ${ }^{49}$ The IRC $^{22}$ was obtained with 460 points by using a step size equal to 0.001 amu ${ }^{1 / 2}$ Bohr. The topological analysis of ELF and the characterization of the associated phase-space portrait at each point incorporating the four type of CPs was performed by using the Multiwfn ${ }^{50}$ and $\mathrm{VMD}^{51}$ programs. For each configuration point along the $I R C{ }^{22}$ the fulfilment of the Poincaré-Hopf theorem has been strictly verified.

\section{Results and discussion}

The DA reaction mechanism between 1,3-budatiene and ethylene reveals seven SSDs separated by 10 catastrophes, as analyzed along the IRC at the chosen level of theory. ${ }^{5 f}$ We will focus entirely on the assignment of the nature of topological bifurcations. The reaction mechanism evolves from SSD-I (1,3butadiene + ethylene) to SSD-VII (cyclohexene), with the transition structure located at SSD-III. Figure 1 reveals representative molecular graphs associated to each SSD including the complete set of CPs. We have labeled the CPs which are directly involved in each bifurcation, including both attractors $\left(a_{i}\right)$ and saddles of index $1\left(s_{i}\right)$.

Two catastrophe bifurcations separate SSD-I and SSD-II and simultaneously emerge in the $C_{3}=C_{4}$ and $C_{5}=C_{6}$ double bonds regions of 1,3-butadiene. A careful scrutiny of the evolution of the topographic maps between the SSD-I and SSD-II (panels a and $\mathrm{b}$ in Figure 1 ) around the first bifurcation state reveals that whereas two maxima of $\eta(\boldsymbol{r} ; \boldsymbol{R}), a_{1}$ and $a_{2}$, remains essentially unaltered, the others attractors $a_{3}$ and $a_{4}$ collide and annihilate with the accompanying saddle points of index $1, s_{1}$ and $s_{2}$, respectively, evolving towards two wandering point as a result. Hence, these catastrophes should be categorized as fold ones in the Thom's classification ${ }^{20,23 a}$ and not as cusp as it has been first claimed. ${ }^{5 f}$ Indeed, this fact can be safely verified by following the value of the modulus of the determinant of the Hessian evaluated at each of the implied $C P$ in the vicinity of the bifurcation. Formally, the observed folds are just one type (i.e., $F: 0+1 \rightarrow w p$ ) of the sixth possibilities for bifurcations associated to such a type of elementary catastrophe implying wandering points (wp). 


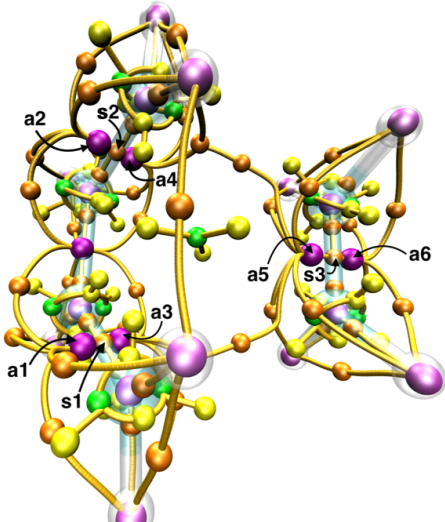

(a) SSD-I

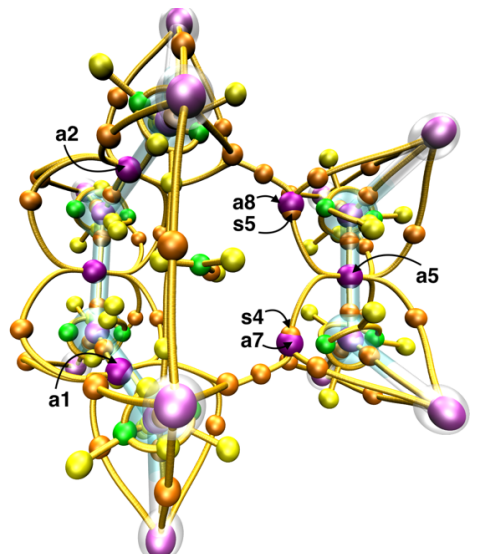

(d) SSD-IV

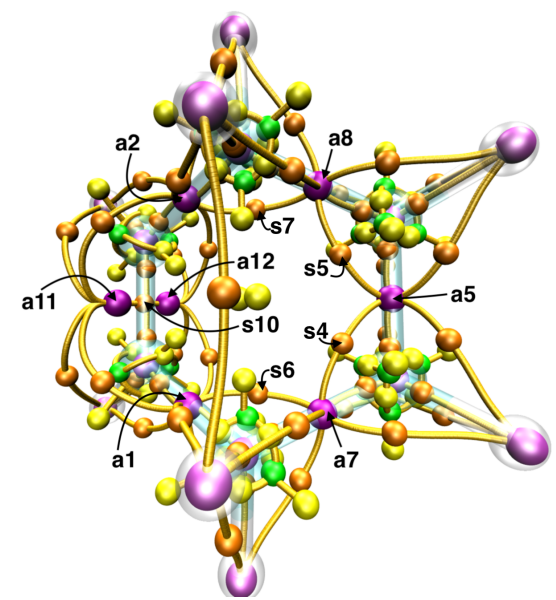

(g) SSD-VII

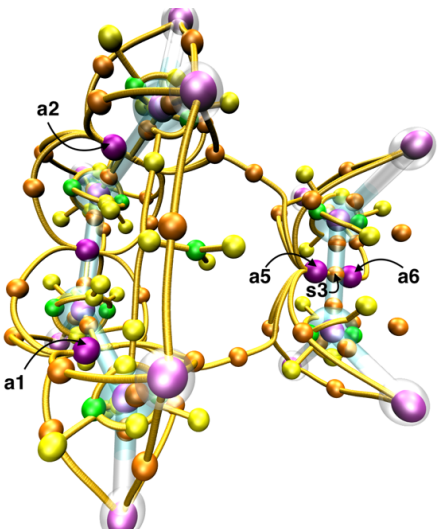

(b) SSD-II

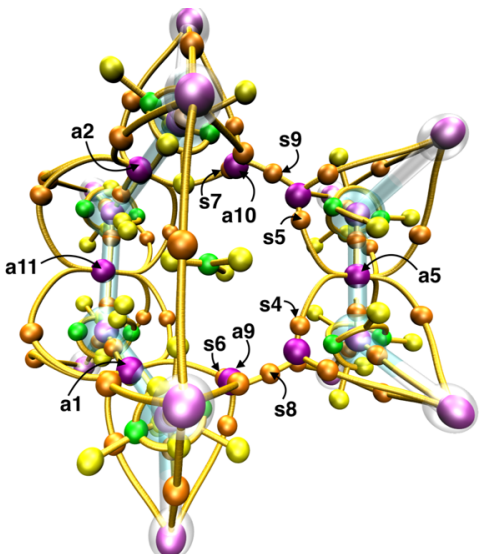

(e) SSD-V

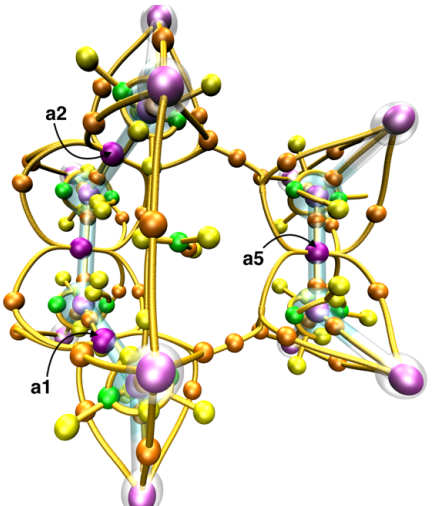

(c) SSD-III

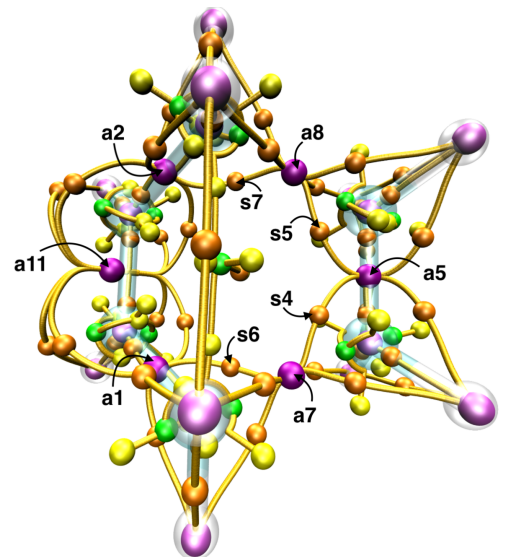

(f) SSD-VI

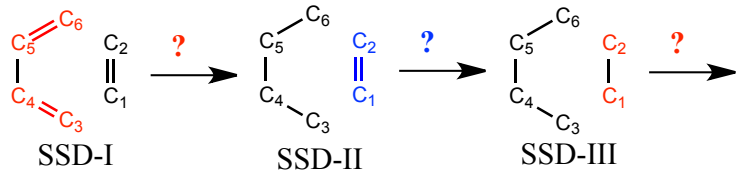

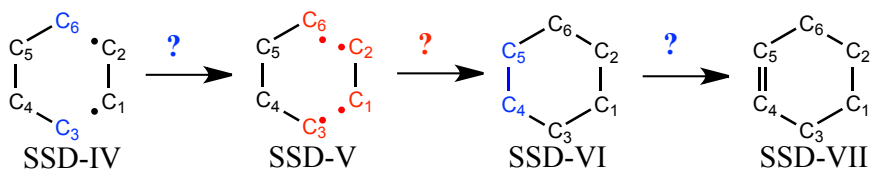

(h)

Figure 1. Molecular graphs associated to each SSD (panels a-g) including all types of CPs of $\eta(\boldsymbol{r} ; \boldsymbol{R})$, i.e., $(3,-3)$ : attractors or maxima of index 0 , represented in purple color; $(3,-1)$ saddles of index 1 , represented in orange color; $(3,+1)$ saddles of index 2 , represented in yellow colour; and $(3,+1)$ repellors or minima of index 3 , represented in green colour. Gradient paths connecting $(3,-3)$ and $(3,-1)$ as well as $(3,+3)$ and $(3,+1)$ are also included. Lewis-type formula representations for each SSD are included for the sake of further clarity (panel h). 


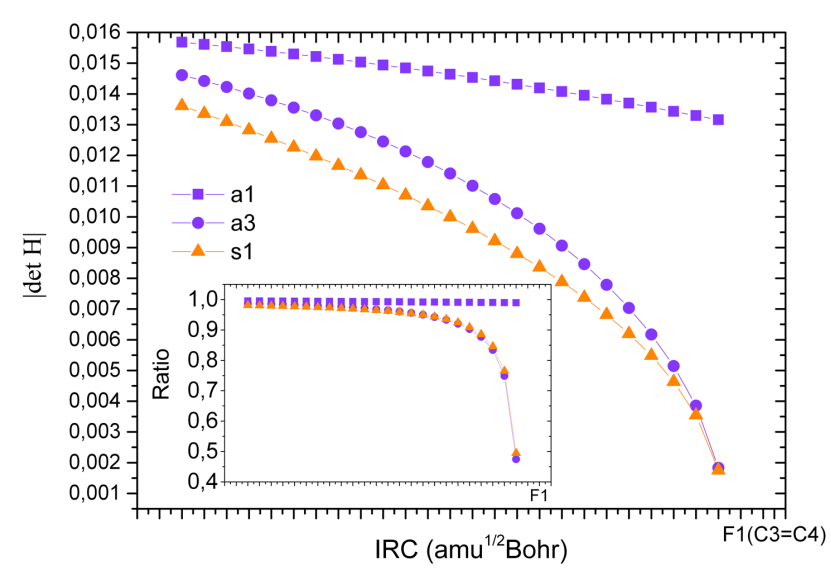

(a)

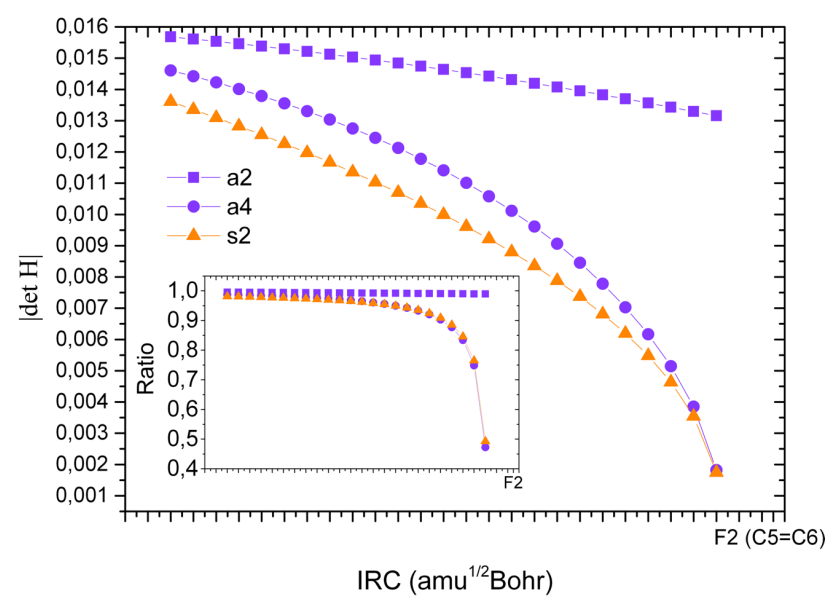

(b)

Figure 2. Variation of the modulus of the determinant of the Hessian matrix of $\eta(\boldsymbol{r} ; \boldsymbol{R})$ evaluated at CPs a1, a3 and s1 (panel a) and a2, a4 and s2 (panel b) for configurations along the SSD-I preceding the concurrent bifurcations F1 and F2 at the CC double bond regions in the 1,3-butadiene moiety. The inner plots reveal the ratio between consecutive values of the $|\operatorname{det} \boldsymbol{H}|$.

Of course, obeying also the necessary condition of fulfilment of the Poincaré-Hopf theorem in any molecular structure with hyperbolic CPS (as those featuring any SSD), the only possibilities for folds are: $F: w p \leftrightarrow 0+1: F^{\dagger}, F: w p \leftrightarrow 0+$ $3: F^{\dagger}$, and $F: w p \leftrightarrow 2+3: F^{\dagger}$, where we use for simplicity just the index of the implied CPs.

In order to illustrate the basis for such an assignment, Figure 2 depicts the variation of the modulus of the determinant of Hessian matrix $|\operatorname{det} \boldsymbol{H}|$ evaluated at the four $(3,-3)$ and the two $(3,-1)$ CPs for 25 sequential configurations along the IRC before bifurcations take place.

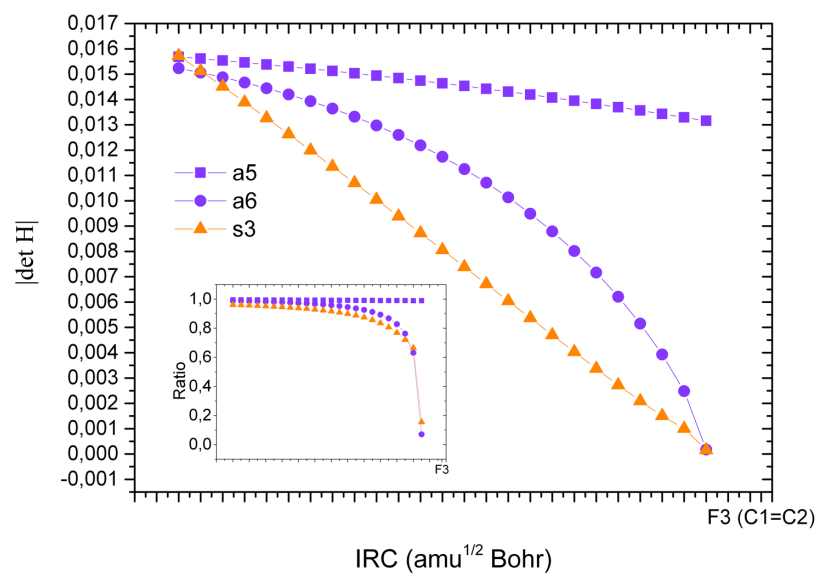

Figure 3. Variation of the modulus of the determinant of the Hessian matrix of $\eta(\boldsymbol{r} ; \boldsymbol{R})$ evaluated at the CPs $a_{5}, a_{6}$ and $s_{3}$ for configurations along the SSD-II preceding the bifurcation $F 3$ located at the $C_{1}=C_{2}$ double bond region in the ethylene moiety. The inner plot reveals the ratio between consecutive values of the $|\operatorname{det} \boldsymbol{H}|$.

The evolution of the variation of $|\operatorname{det} \boldsymbol{H}|$ provides a clear way for determining the CPs involved in the catastrophes and help us to uniquely establish a flag of the degeneration. Just before the bifurcations, the $|\operatorname{det} H|$ remains essentially constant for attractors $a_{1}$ and $a_{2}$, showing a drop by a factor of 0.99 for both $\mathrm{CPs}$, whereas it decreases by a factor of about 2 for $a_{3}, a_{4}, s_{1}$ and $s_{2}$.

The examination of the relative distances between the attractors and saddles involved in the two first catastrophes evidences that distances $a_{3}-s_{1}$ and $a_{4}-s_{2}$ are decreased by a factor of 2.06 each (i.e., varying from $0.0563 \AA$ to $0.0273 \AA$ ), whereas the $a_{1}-s_{1}$ and $a_{2}-s_{4}$ slightly increase by a factor of 1.04 each (i.e., varying from $0.2449 \AA$ to $0.2559 \AA$ ), by considering two configurations along the IRC the concurrent F1 and F2 bifurcations emerges. This suggests that in evolving towards the bifurcation states $\mathrm{F} 1$ and $\mathrm{F} 2$, attractors $a_{3}$ and $a_{4}$ are moving fast in collision with saddles $s_{1}$ and $s_{2}$, respectively, whilst the latter moved in opposite directions with respect to $a_{1}$ and $a_{2}$, respectively. Hence, both the evolution of $|\operatorname{det} \boldsymbol{H}|$ and the relative mobility of these six CPs indicate that maxima $a_{1}$ and $a_{2}$ cannot be linked to the observed bifurcations. Therefore, the concurrent topographical changes associated to the region of double bonds, $C_{3}=C_{4}$ and $C_{5}=C_{6}$, properly correspond to catastrophes of the fold type in which one attractor and one saddle collide in each case to yield a wandering point. No signature of cusp catastrophes could be found.

The third topological bifurcation (separating SSD-II and SSD-III, panels $\mathrm{b}$ and $\mathrm{c}$ in Figure 1, respectively) is associated to the $\mathrm{C}_{1}=\mathrm{C}_{2}$ double bond region in the ethylene fragment. 


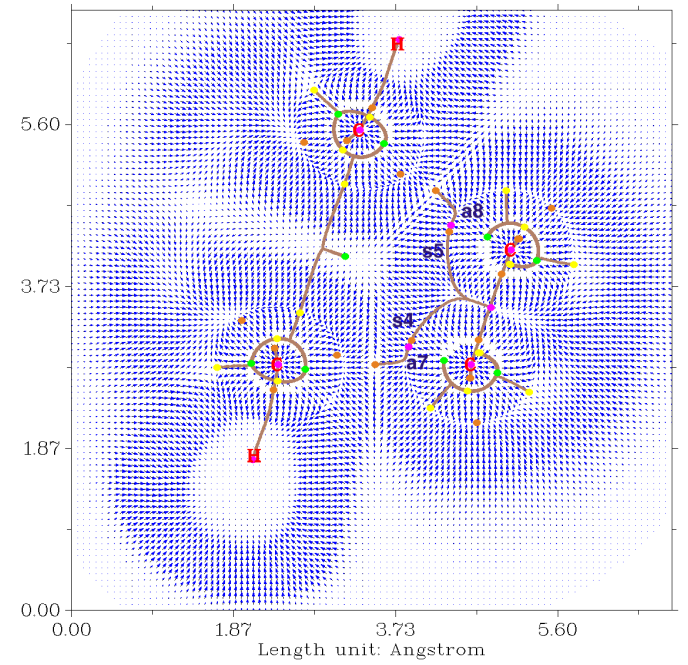

(a)

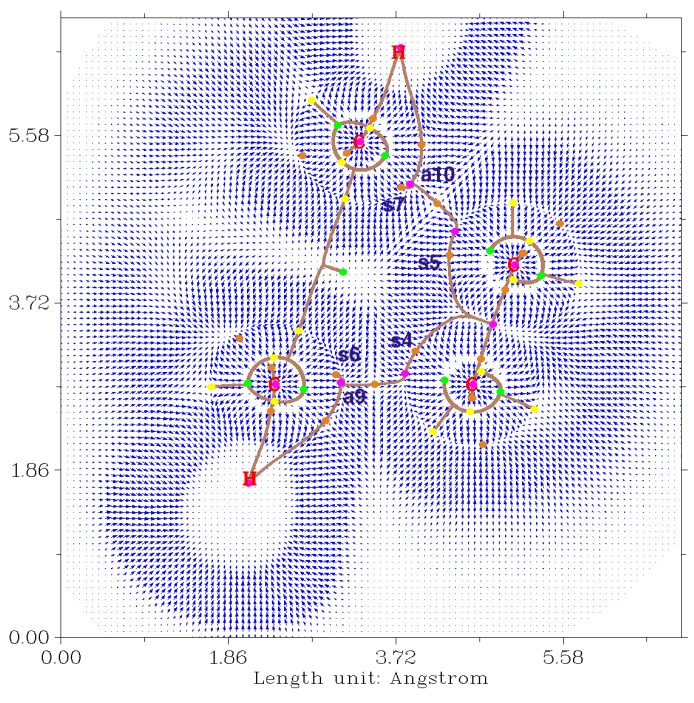

(c)

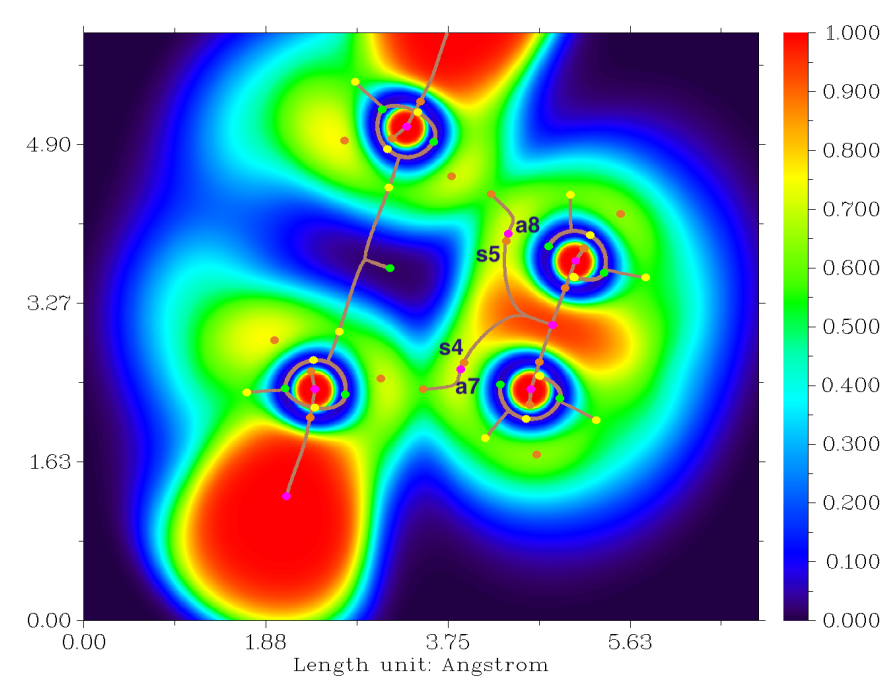

(b)

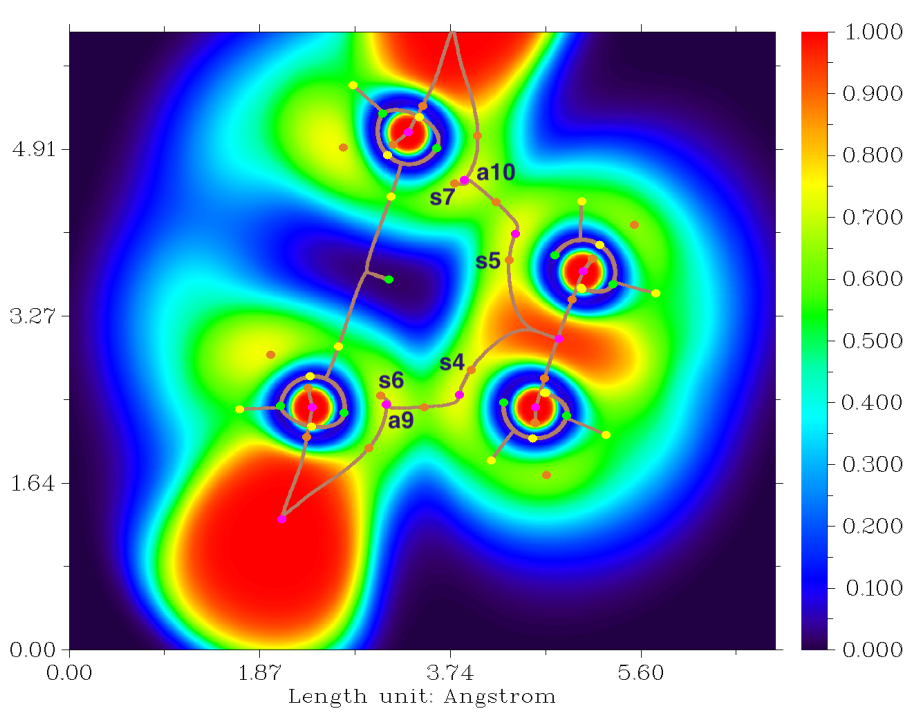

(d)

Figure 4. Vector field ( $\mathrm{a}$ and $\mathrm{c}$ panels), and colour-filled ( $\mathrm{b}$ and $\mathrm{d}$ panels) maps of the electron localization function $\eta$, represented in the plane containing the $\mathrm{C} 1$, $\mathrm{C} 2$, $\mathrm{C} 3$, and $\mathrm{C} 6$ centers for the reaction of 1,3-butadiene and ethylene, just after the fourth and fifth catastrophes ( $\mathrm{a}$ and $\mathrm{b}$ panels) and the sixth and seventh bifurcations ( $\mathrm{c}$ and $\mathrm{d}$ panels). All these catastrophes are of the fold type and are associated to the concurrent emerging of the $(3,-3)$ and $(3,-1)$ CPs in the neighborhood of the carbon centres, namely: at $\mathrm{C} 1$ (i.e., a7 and $\mathrm{s} 4)$ and $\mathrm{C} 2$ (i.e., a8 and s5); and at C3 (i.e., a9 and s6), and C6 (i.e., a10 and s7) occurring within "mixing" or conflicting regions.

The variation of the modulus of the determinant of Hessian matrix, evaluated at the two attractors and the one saddle of index 1 for 25 points along the IRC before the third bifurcation $\mathrm{F} 3$ take place, is presented in Figure 3. For the maximum $a_{6}$ and saddle $s_{3}$ the $|\operatorname{det} \boldsymbol{H}|$ decreases by factors of 14.10 and 6.39 , respectively, whereas it remains almost constant for the attractor $a_{5}$, exhibiting a drop by a factor of 1.01.

The observed pattern of relative distances between the CPs $a_{6}-$ $s_{3}$ at two configurations along the IRC before F3 take place reveals a decreasing by a factor about 9 (i.e., varying from $0.0864 \AA$ to $0.0097 \AA$ ) , whereas the distance $a_{5}-s_{3}$ is indeed increased by a factor of 1.45 (i.e., from $0.0475 \AA$ to $0.0687 \AA$ ).
This fact suggests that attractor $a_{6}$ is moving remarkably faster towards the saddle, whereas $a_{5}$ moves in the opposite direction just before the bifurcation emerges. One should safely describe the change of the topographic map at $C_{1}=C_{2}$ double bond of ethylene by stating that the maximum $a_{6}$ collided with the saddle $s_{3}$ and both CPs are annihilated as a direct consequence of such process. Henceforth, since the attractor $a_{5}$ is not involved in the third bifurcation, the qualitative change in the phase-portrait of the ELF should be associated with a fold elementary catastrophe, again one of the $F: 0+1 \rightarrow w p$ type. 
These findings stress that no cusp catastrophes are associated to the "activation" of the double bonds in the first stages of the DA reaction between butadiene and ethylene. Such an activation is related to the mutual polarization that approaching reagents introduce one each. These changes will yield immediately to concentration of the nonbonding electron density in the neighborhood regions associated to the terminal carbons in both interacting species. In the first place we observe the fourth and fifth catastrophes (separating SSD-III and SSDIV, panels $c$ and $d$ in Figure 1, respectively) which take place simultaneously at the neighborhood of the carbons in the ethylene fragment. Then, the sixth and seventh catastrophes (separating SSD-IV and SSD-V, panels $d$ and e in Figure 1, respectively) which take place simultaneously at the neighborhood of the terminal carbons in the 1,3-butadiene fragment. All these catastrophes correspond indeed to the type $F^{\dagger}: w p \rightarrow 0+1$ as discussed earlier. ${ }^{5 f}$ Complementarily to such an analysis, these bifurcations should be properly described by considering that the flow, generated by solving $\nabla_{r} \eta(\boldsymbol{r} ; \boldsymbol{R})=0$, pushes a regular point of $\eta(\boldsymbol{r} ; \boldsymbol{R})$ into the basin of an attractor passing through a region where lines representing the field are oriented in opposite directions. Therefore, from a formal mathematical point of view the so-called wandering point can now be seen as a CP which degenerates given the instability that characterizes the "mixing" or conflicting region through which it moves. The transversal intersection of the space constructed over the chosen control subspace with that one structured from the so-called bifurcation set, forces the degenerate $\mathrm{CP}$ to break into two hyperbolic CPs (e.g., an attractor and a saddle in the present case, i.e., $a_{7}$ and $s_{4}$ at $C_{1}$, $a_{8}$ and $s_{5}$ at $C_{2}$ in the fourth and fifth concurrent catastrophes, and $a_{9}$ and $s_{6}$ at $C_{3}$, and $a_{10}$ and $s_{7}$ at $C_{6}$, in the case of the sixth and seventh simultaneous bifurcations, see Figure 4). The changes of the phase-space portrait by virtue of an increasing in the number of CPs can be therefore traced back to the transversality condition. ${ }^{20,23}$ Hence, these bifurcations can be naturally associated with the fold elementary catastrophe since the above arguments should be hold in order to properly describe both bifurcations. Commonly, the "scale of time" (in a way of speaking) for the described process is too small to be captured by any a posteriori methodology as traditionally employed in practical applications of BET.5a, 9, 24, 27-40 We recall here that all changes in a topologically defined molecular structure should be always traced back to the emergence of non-Morse or degenerate critical points (in $\mathbb{R}^{3}$, those CPs where the rank of the Hessian matrix is equal to three). It is because these points, which are unstable in the local potential under study with respect to the changes induced by external factors (e.g., nuclei displacements) cause the system evolves towards stable configurations, i.e., with no degenerate or hyperbolic critical points only. ${ }^{20,23}$

The concurrent eighth and ninth catastrophes separate the SSD-V and SSD-VI (panels e and $\mathrm{f}$ in Figure 1, respectively), are associated to the topological changes in the new CC bonds forming regions. The variation of the modulus of the determinant of Hessian matrix for attractors a7, a8, a9 and a10 as well as for the saddles s8 and s9 is revealed in Figure 5. It should be noted that the s8 and s9 CPs are present along the precedent SSDs I-VI. The $|\operatorname{det} \boldsymbol{H}|$ for a9, a10, s8 and s9 decreases by a factor ranging from 1.59 to 1.68 , whereas for maxima $a 7$ and a 8 it exhibits a drop by a factor of 1.30 for both CPs.

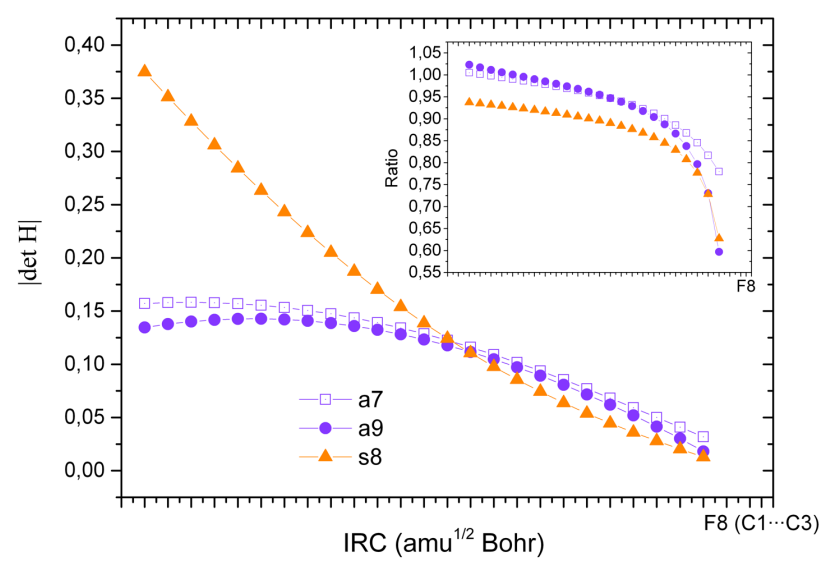

(a)

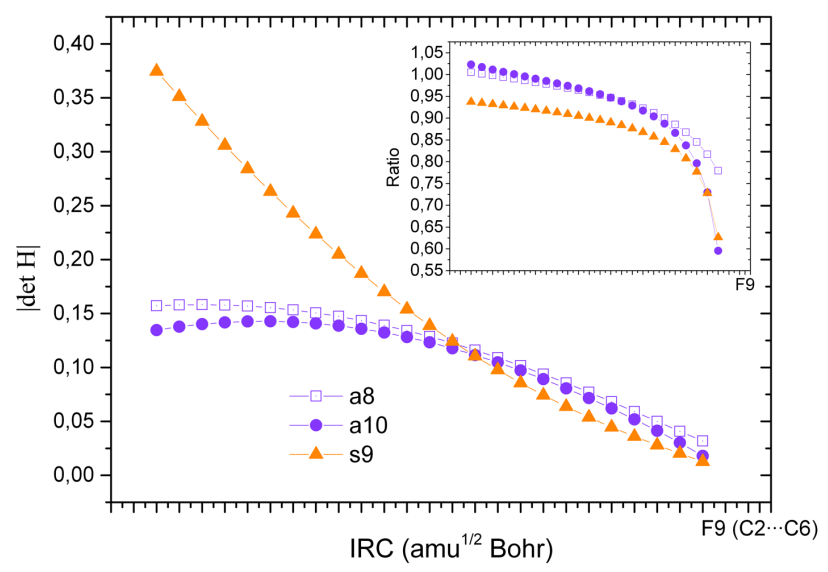

(b)

Figure 5. Variation of the absolute value of the determinant of the Hessian matrix evaluated at the CPs $a_{7}, a_{9}$, and $s_{8}$ (left, a) and at the critical points $a_{8}, a_{10}$ and $s_{9}$ (right, b) for points along the IRC before the eighth and ninth bifurcations occurred at the region of the new $\mathrm{C}-\mathrm{C}$ bond forming. The inner plots reveal the ratio between consecutive values of the $|\operatorname{det} \boldsymbol{H}|$.

Moreover, before the bifurcations the curves of the $|\operatorname{det} \boldsymbol{H}|$ for the maxima tends to be parallel, but then they exhibit a crossover which suggest that as the occurrence of the bifurcations approach the rate of decreasing of the determinant of the Hessian for a9 and $a 10$ is faster than that the one for a7 and $\mathrm{a} 8$, respectively.

Indeed, the examination of the relative distances between the attractors and saddles involved in the two first catastrophes evidences a decreasing by a factor of 1.05 of distances a7-s8 and a8-s9 each (i.e., varying from $0.1596 \AA$ to $0.1519 \AA$ ) , whereas the a9-s8 and a10-s9 decreases by a factor of 1.39 each (i.e., varying from $0.1131 \AA$ to $0.0816 \AA$ ), by considering two configurations along the IRC before F8 and F9 bifurcations. 
bifurcations should be properly described in terms of two simultaneous folds, and not in terms of cusps types as previously remarked. ${ }^{5 f}$

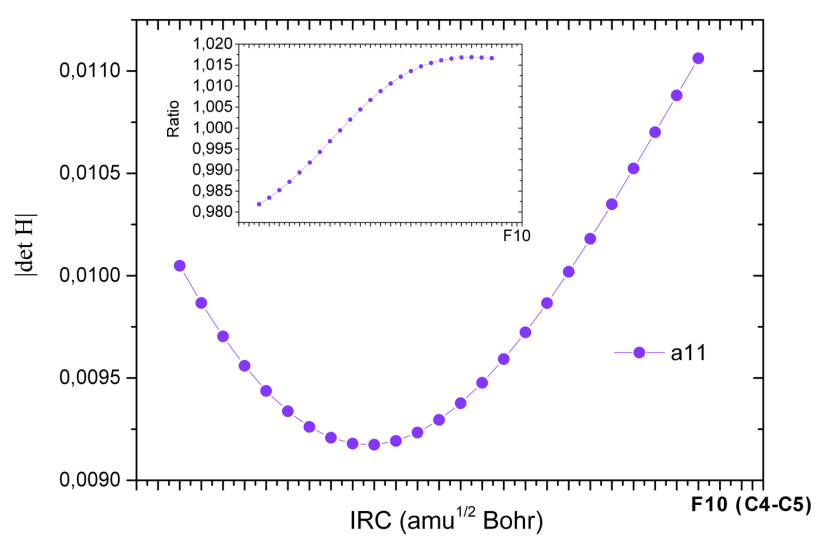

Figure 7. Variation of the modulus of the determinant of the Hessian matrix of $\eta(\boldsymbol{r} ; \boldsymbol{R})$ evaluated at the critical points $a_{11}$ for configurations along the SSD-VI before the bifurcation occurs at the $C_{4}-C_{5}$ bond region. The inner plot reveals the ratio between consecutive values of the $|\operatorname{det} \boldsymbol{H}|$.

(a)

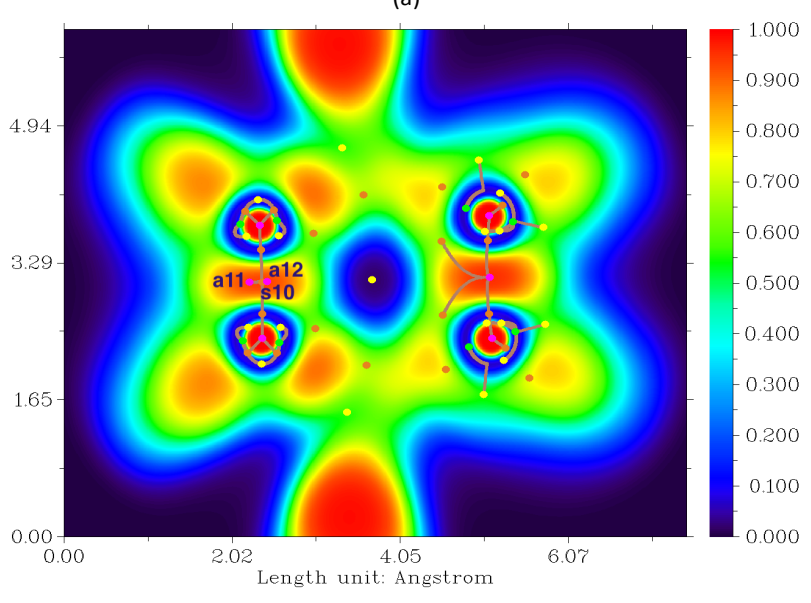

(b)

Figure 6. Vector field (panel a), and colour-filled (panel b) maps of the electron localization function $\eta$, represented in the plane containing the $C_{1}, C_{2}, C_{4}$, and $C_{5}$ carbon centres for the reaction of 1,3-butadiene and ethylene, just after the tenth catastrophe (F10) occurring within a "mixing" or conflicting region located at the $\mathrm{C}_{4}-\mathrm{C}_{5}$ bonding region. It corresponds to fold bifurcation associated to the concurrent creation of CPs or index $0\left(a_{12}\right)$ and $1\left(s_{10}\right)$ from a wandering point.

Thus, based on the behavior of the $|\operatorname{det} \boldsymbol{H}|$ and relative distances between the CPs in the neighborhood of bifurcations, we can establish that $a_{9}$ and $a_{10}$ as well as saddles $s_{8}$ and $s_{9}$ exhibit a clear tendency to degenerate, whereas this is not the case for $a_{7}$ and $a_{8}$. Furthermore, the magnitude of the difference of the relative distance indicates that $a_{9}$ and $a_{10}$ tend to collide with $s_{8}$ and $s_{9}$, respectively, since the former points move towards the corresponding saddle faster in comparison to the observed behavior for attractors $a_{7}$ and $a_{8}$. This suggests that maxima $a_{7}$ and $a_{8}$ must not be linked to the bifurcations, and
The last topological bifurcation (tenth) is related to the topological change located at the CC bonding region between carbons $C_{4}$ and $C_{5}$ along the path yielding the formation of cyclohexene. F10 is associated to the emerging of both the attractor $a_{12}$ and the saddle $s_{10}$ of index equal to 1 in the neighborhood region of the maximum $a_{11}$. This event can be described by the same arguments given for bifurcations emerging from wandering points (catastrophes fourth and fifth), now revealed for F10 in Figure 6. The behavior of the $|\operatorname{det} \boldsymbol{H}|$ for $a_{11}$ is revealed in Figure 7. Note that preceding the tenth bifurcation, it exhibits indeed a pronounced increase. Just after the bifurcation took place, the relative distance between the CPs $a_{11}$ and $s_{10}$ was about 37 times greater than that one between the latter point and $a_{12}$. It is clear that the attractor $a_{11}$ exhibits no tendency to degenerate, being in fact located quite far from the saddle $s_{10}$. These results indicate that the attractor $a_{11}$ is not involved in the topological bifurcation, and the hyperbolic CPs $a_{12}$ and $s_{10}$ must come from a wandering point that became a degenerate $\mathrm{CP}$ and eventually unfolded. These facts emphasize that the bifurcation tenth must be associated to a fold elementary catastrophe, namely, $F^{\dagger}: w p \rightarrow 0+1$.

\section{Conclusions}

The assignment of the nature of elementary catastrophes featuring the Diels-Alder reaction mechanism between 1,3butadiene and ethylene has been re-examined in the present work. On the basis of both the evolution of the determinant of the Hessian matrix evaluated at the involved critical points as well as its associated relative distances, we provide strong support for an affirmative answer to the title question, namely, that only fold catastrophes are implied in the process. Such a methodological framework enables to clarify in an 
unambiguous way the nature of each catastrophe, allowing an adequate characterization that is coherent with all formalities behind the theory. Surprisingly, no flags for cusp bifurcations has been detected along the intrinsic reaction path, even to those associated to the region where the two new CC bonds are formed. Regarding chemically relevant events, the mechanism can be henceforth claimed to be rationalized in terms of seven structural stability domains separated by a sequence of 10 elementary catastrophes of fold $\left(\mathrm{F}\right.$ and $\left.\mathrm{F}^{+}\right)$type, i.e., $\mathrm{C}_{4} \mathrm{H}_{6}+\mathrm{C}_{2} \mathrm{H}_{4}$ : 1-7-[FF]F[F $\left.F^{\dagger} F^{\dagger}\right]\left[F^{\dagger} F^{\dagger}\right][F F] F^{\dagger}-0: C_{6} H_{10}$, where 8 bifurcations occurs simultaneously. It is certainly remarkable all the forming/breaking processes of the chemical bond along this cornerstone reaction mechanism can be associated to the simplest unfolding within the framework of elementary catastrophe theory. ${ }^{20,23}$ It stresses that main bonding changes along the reaction coordinate can be properly studied by using the simplest of the Thom's catastrophe functions, namely, the 1-(state) variable, 1-(control) parameter function. That is, in the neighborhood of a degenerate critical point around the bifurcation configuration, the local character of the electron localization function can be studied in terms of the canonical decomposition associated to the fold function, i.e., $\eta \doteq\left(x^{3}+\right.$ $u x)+\left(\lambda_{2} y_{2}^{2}+\lambda_{3} y_{3}^{2}\right) .{ }^{20}, 23 a, 41$ Here, $x$ stands for the only coordinate associated to the zero eigenvalue of the Hessian matrix, whereas the last Morse part is written in terms of the non-zero eigenvalues $\left(\lambda_{i}\right)$ and associated directions $\left(y_{i}\right)$, after the required smooth changes of variables. ${ }^{23 \mathrm{~b}}$ In the present case of the DA reaction, the control parameter $u$ can always be chosen as the interatomic distance between the implied carbon centers. These surprising findings suggest that earlier conclusions draw from simplified methodological frameworks will require careful reexaminations. We hope these results can further stimulate the discussion of research concerning the detailed identification, characterization, and analysis of chemical processes and reactivity within the methodological framework that offers bonding evolution theory framework ${ }^{21}$ based on the combined used of topological analysis of ELF and catastrophe theory.

\section{Author Contributions}

L. A. and M.D-N performed the computational studies and developed the theoretical, along with their mathematical expressions. C.G., E.R. and P. P. participated in data analyses. E.C. and P.P conceptualized and supervised the study and wrote the manuscript with L.A.

\section{Conflicts of interest}

There are no conflicts to declare.

\section{Acknowledgements}

Authors thank the ANID/CONICYT PhD scholarship awarded to L. Ayarde. We also are indebted to the Fondo Nacional de Ciencia y Tecnología (FONDECYT-ANID, Chile), by the continuous support provided through Projects Nos. 1181582 (EC), and 1180348 (PP).

\section{Notes and references}

1 a) The Nobel Prize in Chemistry 1950 was awarded jointly to Otto Paul Hermann Diels and Kurt Alder "for their discovery and development of the diene synthesis." The Nobel Foundation. $<$ https://www.nobelprize.org/prizes/chemistry/1950/su mmary/>). b) O. Diels and K. Alder, Justus Liebigs Annalen der Chemie, 1928, 460, 98-122.

2 a) M. A. Ischay, Z. Lu and T. P. Yoon, J. Am. Chem. Soc., 2010, 132, 8572-+. b) J. Mahatthananchai, A. M. Dumas and J. W. Bode, Angew. Chem.-Int. Edit., 2012, 51, 1095410990. c) F. M. Bickelhaupt and K. N. Houk, Angew. Chem.-Int. Edit., 2017, 56, 10070-10086. d) S. Shaik, D. Mandal and R. Ramanan, Nat. Chem., 2016, 8, 1091-1098. e) A. Mavroskoufis, K. Rajes, P. Golz, A. Agrawal, V. Russ, J. P. Gotze and M. N. Hopkinson, Angew. Chem.-Int. Edit., 2020, 59, 3190-3194. f) W. Cullen, M. C. Misuraca, C. A. Hunter, N. H. Williams and M. D. Ward, Nat. Chem., 2016, 8, 231-236.

3 a) K. N. Houk, J. Gonzalez and Y. Li, Accounts Chem. Res., $1995,28,81-90$. b) O. Wiest, D. C. Montiel and K. N. Houk, J. Phys. Chem. A, 1997, 101, 8378-8388.

4 a) L. R. Domingo, M. J. Aurell, P. Perez and R. Contreras, Tetrahedron, 2002, 58, 4417-4423. b) H. J. Jiao and P. V. Schleyer, J. Phys. Org. Chem., 1998, 11, 655-662. c) M. J. S. Dewar and C. X. Jie, Accounts Chem. Res., 1992, 25, 537-543. d) B. R. Beno, K. N. Houk and D. A. Singleton, J. Am. Chem. Soc., 1996, 118, 9984-9985.

5 a) E. Chamorro, M. Duque-Noreña, N. Gutierrez-Sánchez, E. Rincón and L. R. Domingo, Journal of Organic Chemistry, 2020, 85, 6675-6686. b) L. R. Domingo, M. Rios-Gutierrez, B. Silvi and P. Perez, Eur. J. Org. Chem., $2018,2018,1107-1120$. c) L. R. Domingo, M. RiosGutierrez, E. Chamorro and P. Perez, ChemistrySelect, 2016, 1, 6026-6039. d) L. R. Domingo and J. A. Saez, Org. Biomol. Chem., 2009, 7, 3576-3583. e) S. Berski, J. Andres, B. Silvi and L. R. Domingo, J. Phys. Chem. A, 2006, 110, 13939-13947. f) S. Berski, J. Andres, B. Silvi and L. R. Domingo, J. Phys. Chem. A, 2003, 107, 6014-6024. g) L. R. Domingo, E. Chamorro and P. Perez, Org. Biomol. Chem., 2010, 8, 5495-5504.

6 a) L. R. Domingo, M. Rios-Gutierrez, P. Perez and E. Chamorro, Mol. Phys., 2016, 114, 1374-1391. b) L. R. Domingo, E. Chamorro and P. Perez, Lett. Org. Chem., 2010, 7, 432-439. c) G. Bentabed-Ababsa, A. Derdour, T. Roisnel, J. A. Saez, P. Perez, E. Chamorro, L. R. Domingo and F. Mongin, Journal of Organic Chemistry, 2009, 74, 2120-2133.

7 a) D. H. Ess and K. N. Houk, J. Am. Chem. Soc., 2008, 130, 10187-10198. b) K. N. Houk, B. R. Beno, M. Nendel, K. Black, H. Y. Yoo, S. Wilsey and J. K. Lee, Theochem-J. Mol. Struct., 1997, 398, 169-179. c) R. Hoffmann and R. B. Woodward, J. Am. Chem. Soc., 1965, 87, 2046-2048.

8 a) P. W. Ayers, C. Morell, F. De Proft and P. Geerlings, Chem.-Eur. J., 2007, 13, 8240-8247. b) P. Geerlings and F. De Proft, Phys. Chem. Chem. Phys., 2008, 10, 3028-3042. c) P. Geerlings, P. W. Ayers, A. Toro-Labbe, P. K. Chattaraj and F. De Proft, Accounts Chem. Res., 2012, 45, 683-695.

9 V. Polo, J. Andres, S. Berski, L. R. Domingo and B. Silvi, J. Phys. Chem. A, 2008, 112, 7128-7136.

10 a) P. L. A. Popelier, in Chemical Bond li: 100 Years Old and Getting Stronger, ed. D. M. P. Mingos, Springer, New York, 2016, vol. 170, pp. 71-117. b) R. F. W. Bader, J. 
Hernandez-Trujillo and F. Cortes-Guzman, J. Comput Chem., 2007, 28, 4-14. c) K. Collard and G. G. Hall, Int. J. Quantum Chem., 1977, 12, 623-637. d) R. F. W. Bader, Atoms in Molecules. A Quantum Theory, Oxford University Press Inc., New York, USA, 1994. e) A. Savin, R. Nesper, S. Wengert and T. F. Fassler, Angew. Chem.-Int. Edit., 1997, 36, 1809-1832. f) B. Silvi and A. Savin, Nature, 1994, 371, 683-686.

11 a) R. F. W. Bader, J. Phys. Chem. A, 1998, 102, 7314-7323. b) R. F. W. Bader, Theor. Chem. Acc., 2001, 105, 276-283.

12 A. D. Becke and K. E. Edgecombe, J. Chem. Phys., 1990, 92, 5397-5403.

13 a) L. R. Domingo, E. Chamorro and P. Perez, Journal of Organic Chemistry, 2008, 73, 4615-4624. b) B. Silvi, J. Mol. Struct., 2002, 614, 3-10.

14 a) N. O. J. Malcolm and P. L. A. Popelier, Faraday Discuss., 2003, 124, 353-363. b) R. F. W. Bader and M. E. Stephens, J. Am. Chem. Soc., 1975, 97, 7391-7399.

15 P. L. A. Popelier and E. A. G. Bremond, Int. J. Quantum Chem., 2009, 109, 2542-2553.

16 a) M. Leboeuf, A. M. Koster, K. Jug and D. R. Salahub, J. Chem. Phys., 1999, 111, 4893-4905. b) S. R. Gadre, S. A. Kulkarni and I. H. Shrivastava, J. Chem. Phys., 1992, 96, 5253-5260.

17 T. A. Keith, R. F. W. Bader and Y. Aray, Int. J. Quantum Chem., 1996, 57, 183-198.

18 T. A. Keith and R. F. W. Bader, J. Chem. Phys., 1993, 99, 3669-3682.

19 G. Bruno, G. Macetti, L. Lo Presti and C. Gatti, Molecules, 2020, 25, 26.

20 a) R. Thom, Structural Stability and Morphogenesis: An Outline of a General Theory of Models, Westview Press, 1972. b) T. Poston and I. Stewart, Catastrophe Theory and Its Applications, Pitman Publishin Limited, London. San Francisco. Melbourne, 1978.

21 X. Krokidis, S. Noury and B. Silvi, J. Phys. Chem. A, 1997, 101, 7277-7282.

22 a) K. Fukui, Accounts Chem. Res., 1981, 14, 363-368. b) C. Gonzalez and H. B. Schlegel, J. Chem. Phys., 1991, 95, 5853-5860.

23 a) D. P. L. Castrigiano and S. A. Hayes, Catastrophe Theory, CRC Press. Taylor \& Francis Group. , Boca Raton, FL 33487-2742, Second Edition edn., 2018. b) R. Gilmore, Catastrophe Theory for Scientists and Engineers Dover Publications, 1993.

24 a) J. Andres, P. Gonzalez-Navarrete, V. S. Safont and B. Silvi, Phys. Chem. Chem. Phys., 2017, 19, 29031-29046. b) J. Andres, S. Berski, L. R. Domingo, V. Polo and B. Silvi, Curr. Org. Chem., 2011, 15, 3566-3575. c) J. Andres, L. Gracia, P. Gonzalez-Navarrete and V. S. Safont, Comput. Theor. Chem., 2015, 1053, 17-30. d) J. Andres, P. Gonzalez-Navarrete and V. S. Safont, Int. J. Quantum Chem., 2014, 114, 1239-1252.

25 A. S. Nizovtsev, J. Comput. Chem., 2013, 34, 1917-1924.

26 I. Viciano, P. Gonzalez-Navarrete, J. Andres and S. Marti, J. Chem. Theory Comput., 2015, 11, 1470-1480.

27 P. Gonzalez-Navarrete, L. R. Domingo, J. Andres, S. Berski and B. Silvi, J. Comput. Chem., 2012, 33, 2400-2411.

28 a) A. I. Adjieufack, V. Liegeois, I. N. Mboumbouo, J. K. Mbadcam and B. Champagne, J. Phys. Chem. A, 2018, 122, 7472-7481. b) M. Rios-Gutierrez, P. Perez and L. R. Domingo, RSC Adv., 2015, 5, 58464-58477.

29 a) P. Merino, T. Tejero, I. Delso and R. Matute, Org. Biomol. Chem., 2017, 15, 3364-3375. b) A. I. Adjieufack, I. M. Ndassa, I. Patouossa, J. K. Mbadcam, V. S. Safont, M Oliva and J. Andres, Phys. Chem. Chem. Phys., 2017, 19, 18288-18302.
30 M. Oliva, V. S. Safont, P. Gonzalez-Navarrete and J. Andres, Theor. Chem. Acc., 2017, 136, 11.

31 E. Zahedi, S. Shaabani and A. Shiroudi, J. Phys. Chem. A, 2017, 121, 8504-8517.

32 O. Matthies, Y. Grin and M. Kohout, ChemistrySelect, 2017, 2, 7659-7669.

33 a) A. Cmikiewicz, A. J. Gordon and S. Berski, Struct. Chem., 2018, 29, 243-255. b) S. Berski and L. Z. Ciunik, Mol. Phys., 2015, 113, 765-781.

34 P. Gonzalez-Navarrete, J. Andres and S. Berski, J. Phys. Chem. Lett., 2012, 3, 2500-2505.

35 a) P. Gonzalez-Navarrete, J. Andres and V. S. Safont, Phys. Chem. Chem. Phys., 2018, 20, 535-541. b) A. Nouri, E. Zahedi, M. Ehsani, A. Nouri and E. Balali, J. Sulfur Chem., 2018, 39, 350-366. c) S. Berski and P. Durlak, New J. Chem., 2016, 40, 8717-8726.

36 V. Keley and E. Zahedi, J. Mol. Graph., 2019, 87, 22-29.

37 E. Chamorro, M. Duque-Norena, S. Kaya, E. Rincon and P. Perez, J. Mol. Model., 2018, 24, 12.

38 J. Munarriz, E. Velez, M. A. Casado and V. Polo, Phys. Chem. Chem. Phys., 2018, 20, 1105-1113.

39 A. Nouri, E. Zahedi, M. Ehsani, A. Nouri and E. Balali, Comput. Theor. Chem., 2018, 1130, 121-129.

40 J. Munarriz, R. Laplaza and V. Polo, Mol. Phys., 2019, 117, $1315-1324$

41 V. I. Arnold, V. S. Afrajmovich, Y. S. Ol'yashenko and L. P. Shil'nikov, Bifurcation Theory and Catastrophe Theory, Springer-Verlag, Berlin-Heildelberg, Germany, 1999.

42 A. Savin, A. D. Becke, J. Flad, R. Nesper, H. Preuss and H. G. Vonschnering, Angew. Chem.-Int. Edit., 1991, 30, 409412.

43 a) E. Matito, B. Silvi, M. Duran and M. Sola, J. Chem. Phys., 2006, 125. b) F. Feixas, E. Matito, M. Duran, M. Sola and B. Silvi, J. Chem. Theory Comput., 2010, 6, 2736-2742.

44 B. Maulen, A. Echeverri, T. Gomez, P. Fuentealba and C. Cardenas, J. Chem. Theory Comput., 2019, 15, 55325542.

45 A. Savin, B. Silvi and F. Colonna, Canadian Journal of Chemistry-Revue Canadienne De Chimie, 1996, 74, 10881096.

46 R. S. Palais, Topology, 1963, 2, 299-340.

47 Encyclopaedia of Mathematics, Kluwer Academic Publishers, Dordrecht, 1991.

48 a) M. M. Peixoto, Topology, 1962, 1, 101-120. b) R. Garcia, C. Gutierrez and J. Sotomayor, Bull. Sci. Math., 1999, 123, 599-622.

49 M. J. Frisch, G. W. Trucks, H. B. Schlegel, G. E. Scuseria, M. A. Robb, J. R. Cheeseman, G. Scalmani, V. Barone, B. Mennucci, G. A. Petersson, H. Nakatsuji, M. Caricato, X. Li, H. P. Hratchian, A. F. Izmaylov, J. Bloino, G. Zheng, J. L. Sonnenberg, M. Hada, M. Ehara, K. Toyota, R. Fukuda, J. Hasegawa, M. Ishida, T. Nakajima, Y. Honda, O. Kitao, H. Nakai, T. Vreven, J. Montgomery, J. A., J. E. Peralta, F. Ogliaro, M. Bearpark, J. J. Heyd, E. Brothers, K. N. Kudin, V. N. Staroverov, T. Keith, R. Kobayashi, J. Normand, K. Raghavachari, A. Rendell, J. C. Burant, S. S. Iyengar, J. Tomasi, M. Cossi, N. Rega, J. M. Millam, M. Klene, J. E. Knox, J. B. Cross, V. Bakken, C. Adamo, J. Jaramillo, R. Gomperts, R. E. Stratmann, O. Yazyev, A. J. Austin, R. Cammi, C. Pomelli, J. W. Ochterski, R. L. Martin, K. Morokuma, V. G. Zakrzewski, G. A. Voth, P. Salvador, J. J. Dannenberg, S. Dapprich, A. D. Daniels, O. Farkas, J. B. Foresman, J. V. Ortiz, J. Cioslowski and D. J. Fox, Journal, 2013.

50 T. Lu and F. W. Chen, J. Comput. Chem., 2012, 33, 580592.

$51 \mathrm{~W}$. Humphrey, A. Dalke and K. Schulten, Journal of Molecular Graphics 1996, 14, 33-38. 
Table of Content Graphics

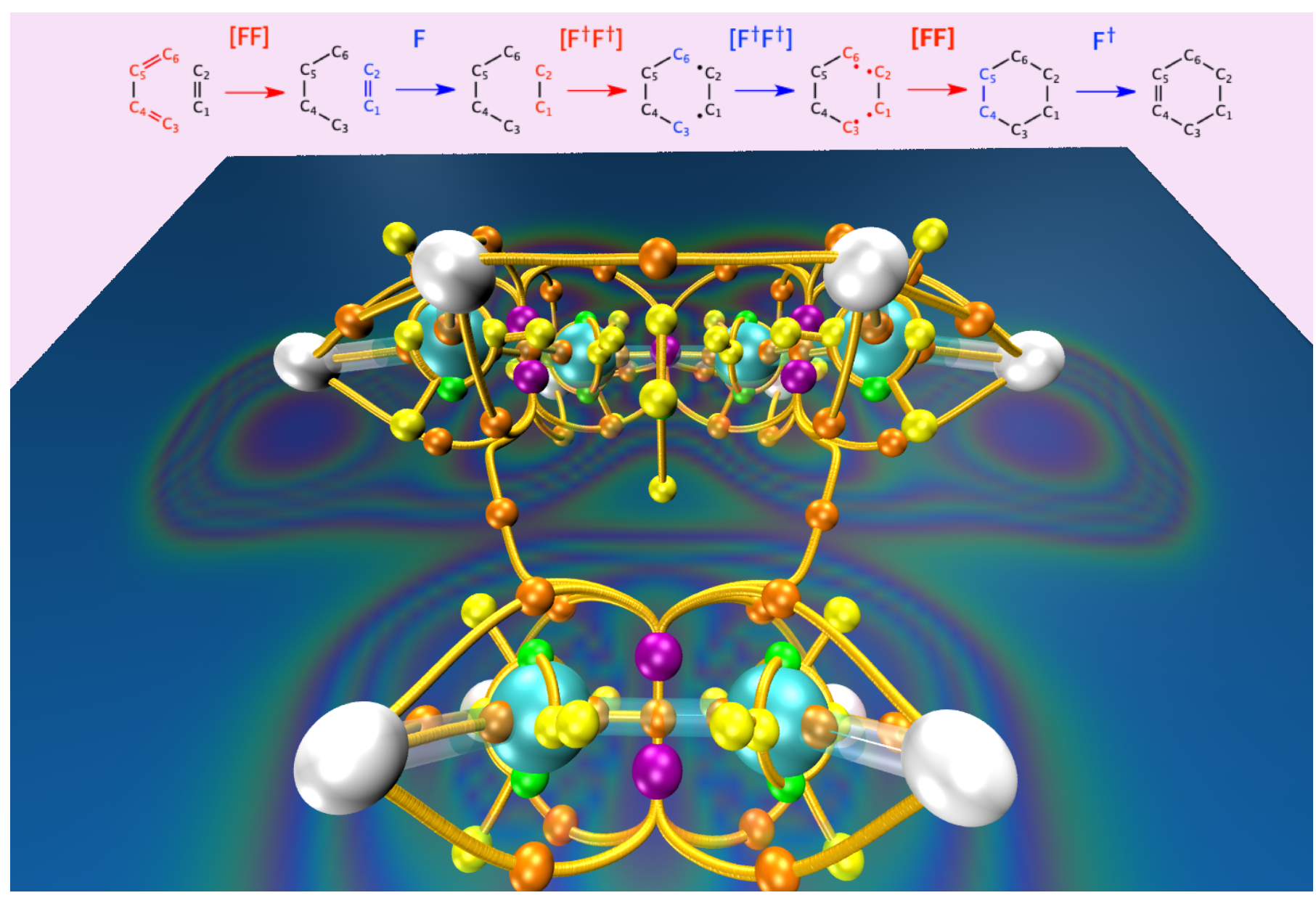

